\title{
Efficacy of Artemether-Lumefantrine for the Treatment of Plasmodium falciparum Malaria in Bohicon and Kandi, Republic of Benin, 2018-2019
}

\author{
Augustin Kpemasse, ${ }^{1}$ Fortune Dagnon, ${ }^{2}$ Ramani Saliou, ${ }^{1}$ Alexis Sacca Yarou Maye, ${ }^{1}$ Cyriaque Dossou Affoukou, ${ }^{1}$ \\ Alassane Zoulkaneri, ${ }^{3}$ Blaise Guézo-Mévo, ${ }^{4}$ Leah F. Moriarty, ${ }^{5 *}$ Yaye D. Ndiaye, ${ }^{6}$ Mamane Nassirou Garba, ${ }^{6}$ Awa Bineta Deme,${ }^{6}$ \\ Daouda Ndiaye, ${ }^{6}$ and Aurore Ogouyemi Hounto ${ }^{1}$ \\ ${ }^{1}$ Laboratory Service and Chemo Sensitivity, Benin National Malaria Control Program, Cotonou, Benin; ${ }^{2}$ U.S. President's Malaria Initiative, U.S. \\ Agency for International Development Benin Office, Cotonou, Benin; ${ }^{3}$ Kandi Health Center, Alibori Department, Benin Ministry of Health, Kandi, \\ Benin; ${ }^{4}$ Bohicon Health Center, Zou Department, Benin Ministry of Health, Bohicon, Benin; ${ }^{5}$ Malaria Branch, Division of Parasitic Diseases and \\ Malaria, Centerfor Global Health, Centers for Disease Control and Prevention, and the U.S. President's Malaria Initiative, Atlanta, Georgia; ${ }^{6}$ Molecular \\ Biology and Genomics Laboratory, Aristide Hospital DANTEC, Dakar, Senegal
}

\begin{abstract}
In 2005, artemether-lumefantrine (AL), an artemisinin-based combination therapy, was introduced as the firstline treatment of uncomplicated Plasmodium falciparum malaria in Benin. Per World Health Organization recommendations to monitor the efficacy of antimalarial treatment, we conducted a therapeutic efficacy study with AL for uncomplicated $P$. falciparum malaria in Bohicon and Kandi, Benin, from 2018 to 2019. Febrile patients aged 6 to 59 months with confirmed $P$. falciparum monoinfection received supervised doses of AL for 3 days. We monitored patients clinically and parasitologically on days $1,2,3,7,14,21$, and 28 . A molecular analysis to detect mutations in the $P$. falciparum Kelch propeller gene (Pfk13) gene was carried out on day 0 samples. A total of 205 patients were included in the study. In Bohicon, the uncorrected adequate clinical and parasitological response (ACPR) proportion was 91.3\% (95\% confidence interval [Cl]: 84.6-95.8\%), whereas in Kandi this proportion was 96.7\% (95\% Cl: 90.6-99.3\%). Genotype-corrected ACPR proportions were 96.3\% (95\% Cl: 90.9-99.0\%) and 96.7\% (95\% Cl: 90.6-99.3\%) in Bohicon and Kandi, respectively. On day 3, 100\% of patients in Bohicon and $98.9 \%$ of patients in Kandi had undetectable parasitemia. The C580Y mutation in the Pfk13 gene was not observed. AL remains effective for $P$. falciparum malaria in these two sites in Benin. Monitoring antimalarial efficacy and prevalence of molecular-resistance markers in Benin should be continued to allow for early detection of antimalarial resistance and to guide treatment policies.
\end{abstract}

\section{INTRODUCTION}

Malaria is a major public health problem in Africa and other tropical areas. Approximately 229 million malaria cases and 409,000 related deaths, mostly in children under 5 years of age, occurred worldwide in 2019. ${ }^{1}$ Benin registered approximately 2.2 million malaria cases and 2,251 deaths in 2018, mostly in children under 5 years of age. ${ }^{2}$ Early diagnosis and prompt treatment with effective antimalarials are among the most important strategies for the control and prevention of malaria. Artemisinin-based combination therapy (ACT) is the standard of care to treat Plasmodium falciparum malaria across Africa. ${ }^{3,4}$ Drug resistance can adversely affect efforts to control malaria and lead to increased malaria-related mortality and morbidity. The WHO recommends monitoring of antimalarial efficacy using therapeutic efficacy studies (TESs) every 2 years in areas of high transmission to guide antimalarial treatment policies. ${ }^{5}$ Day 3 parasite clearance and monitoring for molecular markers of antimalarial resistance may be added to TESs as complementary components. ${ }^{6}$

Following international recommendations, Benin introduced artemether-lumefantrine $(A L)$, an $A C T$, for treatment of uncomplicated $P$. falciparum malaria in 2005 as a result of increasing failure with chloroquine and sulphadoxine-pyrimethamine. ${ }^{7}$ The Ministry of Health, through the National Malaria Control Program (NMCP), has been routinely conducting TESs at several sentinel sites located in different parts of the country to monitor treatment response to the first-line

*Address correspondence to Leah F. Moriarty, Malaria Branch, Division of Parasitic Diseases and Malaria, Center for Global Health, Centers for Disease Control and Prevention, and the U.S. President's Malaria Initiative, 1600 Clifton Rd. NE, Atlanta, GA 30329. E-mail: Imoriarty@cdc.gov treatment, with very high observed polymerase chain reaction (PCR)-corrected efficacy findings in different parts of the country. ${ }^{8,9}$ However, efficacy of $A L$ in neighboring countries has been more variable in recent years, with evidence of inadequate efficacy found in some studies. ${ }^{10-12}$ To guide rational antimalarial policy in Benin, a 28-day TES of AL for the treatment of uncomplicated $P$. falciparum malaria was conducted in Bohicon and Kandi. In addition, resistance marker testing was performed to detect mutations in the $P$. falciparum Kelch propeller gene (Pfk13) associated with artemisinin resistance. ${ }^{6}$

\section{METHODS}

Study timing and location. This single-arm prospective study was conducted during the malaria transmission seasons in 2018 and 2019 at Bohicon and Kandi health centers in Zou and Alibori departments, respectively (Figure 1). An assessment of laboratory records and national data revealed that approximately 424 and 305 patients are diagnosed and treated for malaria per month in Bohicon and Kandi, respectively. ${ }^{2}$ Malaria patients diagnosed at these health facilities who met inclusion criteria were offered participation and were enrolled after a parent or guardian signed the study informed consent form. All parents received a copy of this form for their records.

Sample size. Each site was considered an independent sampling unit. The minimum sample size needed per site to detect a $50 \%$ treatment failure proportion with a precision of $10 \%$ and a confidence interval (Cl) of $95 \%$ was 96 . To account for loss to follow-up in the 28-day period, the final sample size was increased by $20 \%$, for a target of 115 patients per site.

Study procedures. Two teams, one per site, were assembled. Each team comprised one doctor and three nurses who were responsible for screening children with malaria, two microscopists who prepared and read thin and thick smears 


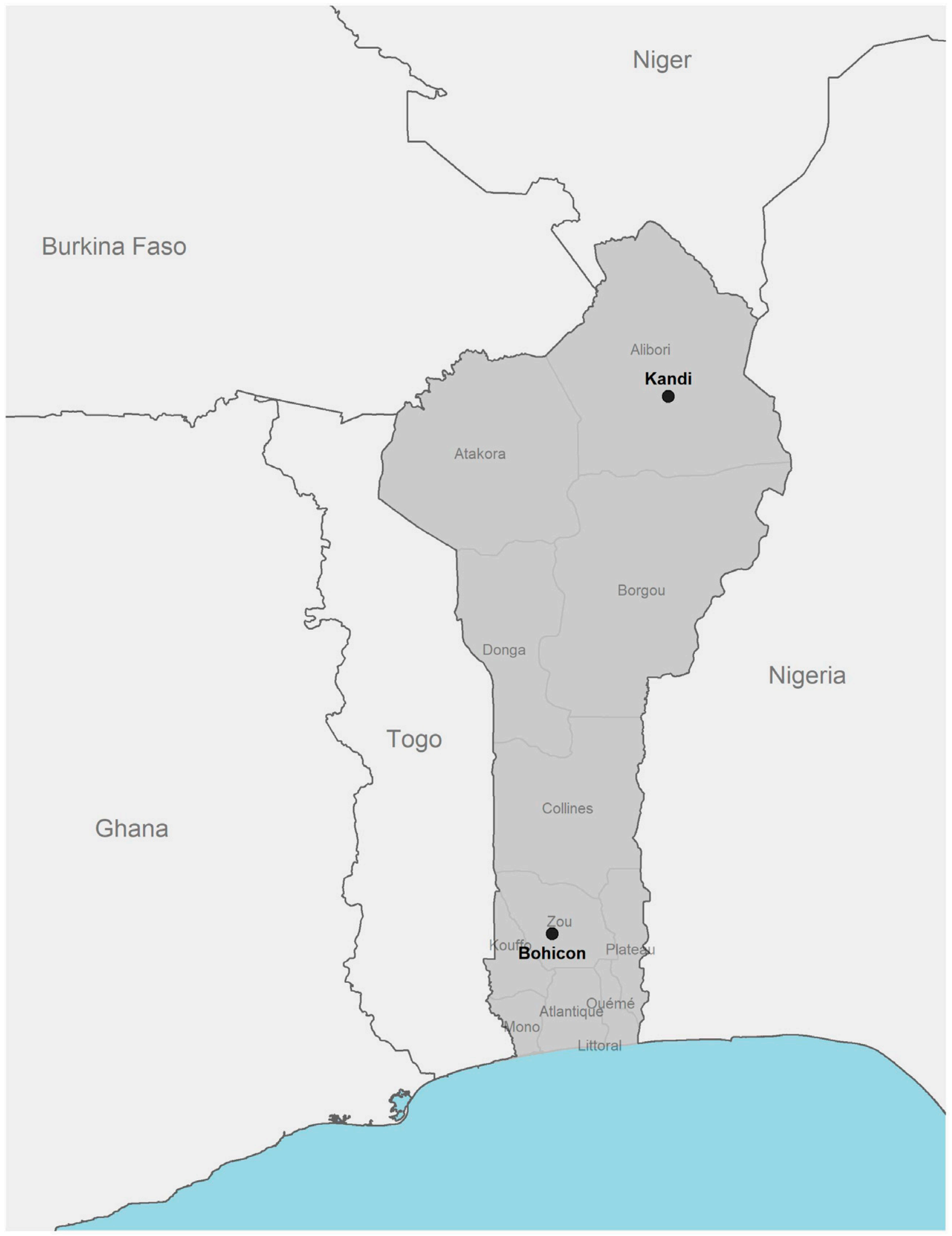

FIGURE 1. Bohicon and Kandi municipalities, sites for antimalarial therapeutic efficacy monitoring, Benin, 2018-2019. This figure appears in color at www.ajtmh.org. 
and other laboratory tests, and a technician to follow up with participants who did not attend scheduled study appointments. Each study team was supervised by a health zone coordinator.

The protocol for this in vivo study was based on the standard WHO methodology for surveillance of antimalarial drug efficacy. ${ }^{5}$ Febrile patients with a malaria blood smear positive for $P$. falciparum monoinfection at the aforementioned study sites were approached by the study team and offered participation. Study inclusion criteria included age $\leq 5$ years, body weight $\geq 5 \mathrm{~kg}$, hemoglobin level $\geq 5 \mathrm{~g} / \mathrm{dL}$, documented fever (axillary temperature $\geq 37.5^{\circ} \mathrm{C}$ ) or recent history of fever, ability to swallow oral medication, parasite density between 2,000 and 200,000 asexual parasites/ $\mu \mathrm{L}$, possibility and willingness of parents or guardians to follow the study protocol, and willingness to return to clinic or accept home visits during the 28-day follow-up. Exclusion criteria included presence of malaria danger signs or signs of severe malaria; history of chronic or severe underlying disease, including malnutrition; allergy to AL; and breastfeeding if mothers were receiving an antimalarial. After obtaining informed consent, demographic and clinical information was collected using standard clinical report forms (CRFs). Patients were seen on day 0 (enrollment), daily from days 1 through 3 , day 7 , and then weekly thereafter until day 28. Patients were also asked to return to clinic if they were ill.

At enrollment, day 0 , three pairs of thick and thin smears were obtained by the study microscopist. One was stained with $10 \%$ Giemsa for fast staining and ascertainment of inclusion criteria. All other smears, including those collected on subsequent visits, were stained with 3\% Giemsa for accurate parasite identification and density estimation. In addition, blood was collected by finger prick for hemoglobin determination (FaStep $\mathrm{HB}^{\circledR}$, AMS Laboratories, Galgorm, United Kingdom) and for preparation of dried blood spots (DBSs) on filter paper (Whatman 903 Protein Saver Cards ${ }^{\circledR}$, Forest Farm Industrial Estate, Cardiff, United Kingdom) for molecular tests.

The AL (20 mg artemether/120 mg lumefantrine) used in the study (Lumiter ${ }^{\circledR}$, Macleods Pharmaceuticals, India) was obtained by the Benin NMCP, which procured the drug after a precertification process. Treatment with $A L$ was administered with a teaspoon of oil and followed manufacturer's weight-based dosing (5-14 kg: 1 tablet per dose, 15-24 kg: 2 tablets, 25-34 kg: 3 tablets) twice a day for 3 days. Participants received all treatment doses under direct observation and were observed for 30 minutes after each dose to monitor for vomiting or other adverse events. Those who vomited were given a second full dose and observed for additional 30 minutes. Inability to tolerate the repeat dose resulted in removal from the study and admission to the hospital for parenteral treatment.

Study teams saw participants for clinical and parasitological evaluation and the administration of the corresponding doses of $A L$ on days 1 and 2 . The day 3 visit was conducted as close to 72 hours from the first dose as possible to accurately assess for delayed parasite clearance. At each follow-up visit, the team administered a standard symptom questionnaire and conducted a physical exam, including measurement of axillary temperature, and blood was collected by finger prick for thick and thin smears, except on day 1 when no smear was collected. In case of parasitemia on day 7 or later, blood samples were collected on filter paper for molecular testing.
Hemoglobin levels were also measured on days 14 and 28 using the same method as for day 0 .

Microscopic blood examination. Before study initiation, two study microscopists per site were trained for 4 days on smear preparation, staining, and microscopy reading and reporting by Benin and National Public Health Laboratory expert microscopists. During monthly supervisory visits, staff from the national laboratory read approximately $10 \%$ of slides for quality control. Parasite density was estimated by counting the number of asexual parasites against 200 white blood cells (WBCs) in the thick smear and estimated in parasites $/ \mu \mathrm{L}$ based on an estimated WBC count of $6,000 \mathrm{WBC} / \mu \mathrm{L}$. When fewer than 100 parasites counted at the 200 WBCs mark, counting continued until 500 WBCs were seen. A total of 1,000 WBCs were counted before a blood smear was considered negative. Smears were read by two independent microscopists blinded to each other; in case of discordance in species determination or if asexual parasite density estimates differed by more than $25 \%$, a third microscopist read the slide. Asexual parasite density was reported as a geometric mean of the two concordant readers. Gametocyte presence was reported as detectable or undetectable.

Study outcomes. The WHO definition of therapeutic responses was used to classify participant outcomes. ${ }^{5}$ In summary, early treatment failure (ETF) was defined as having signs of severe malaria on days $1-3$, asexual parasite density on day 2 higher than on day 0 , parasitemia on day 3 with axillary temperature $\geq 37.5^{\circ} \mathrm{C}$, or parasitemia on day $3 \geq 25 \%$ of day 0 . Late treatment failure was classified as either late clinical failure (LCF) or late parasitological failure (LPF). LCF was defined as parasitemia in the presence of either danger signs of severe malaria or axillary temperature $\geq 37.5^{\circ} \mathrm{C}$ on any day between day 4 and 28 in participants who did not previously meet criteria for ETF. LPF was defined as presence of parasitemia on any day between day 7 and 28 in the absence of fever or any criteria for ETF or LCF. Adequate clinical and parasitological response (ACPR) was defined as absence of parasitemia on day 28 in participants who did not previously meet any of the criteria for ETF, LCF, or LPF. Participants who met criteria for ETF, LCF, or LPF were removed from the study and treated with a rescue regimen (parenteral artesunate, artemether, or quinine) per Benin treatment guidelines. ${ }^{7}$

Molecular testing. To distinguish recrudescences from new infections, genotyping was conducted at the Molecular Biology and Genomics Laboratory at Aristide Hospital DANTEC in Dakar, Senegal. Genomic DNA was extracted from filter paper punches using the manufacturer's protocol for Qiagen DNA Blood kit (Qiagen, Hilden, Germany). First, we performed molecular diagnostic confirmation using nested PCR for day 0 and day of recurrence samples to confirm $P$. falciparum infection. ${ }^{13,14}$ Then, to differentiate recurrences into reinfections and recrudescences, genotyping was performed using primers to the central block of the merozoite surface proteins 2 ( $m s p 2$ ), the glutamate rich protein (glurp), and the block 2 of the merozoite surface proteins 1 (msp1) genes, in that order. ${ }^{15}$ Amplification products were separately on a $2 \%$ agarose gel impregnated with ethidium bromide and visualized under ultraviolet light. If paired samples did not have at least one match, that is, a band with the exact same size upon gel examination, for a given gene, the subsequent amplifications were not conducted, and the paired samples were considered heterologous and associated recurrence deemed reinfection 
in the genotype-corrected analysis. Recurrences for which paired samples shared at least one size-matched band for each one of the three genes were considered recrudescences in the genotype-corrected analysis.

We used the high-resolution melting ( $\mathrm{HRM}$ ) technique to genotype the C580Y mutation of the Pfk13 gene of all day 0 samples. ${ }^{16}$ Two DNA reference samples (NF54 transgenic line with 580Y mutant allele and the clone 7 wild-type C580) were used to detect the polymorphism. Study samples were diluted to $10 \mathrm{ng} / \mu \mathrm{L}$ in standard grade water. We added working stock solution with forward and reverse primers and probes, HRM Light Scanner ${ }^{\circledR}$ Master Mix (Idaho Technology Inc., Salt Lake City, UT), and PCR-grade water to DNA template for a total reaction volume of $10 \mu \mathrm{L}$. The reaction was run on the Light Cycler 96 (Roche Molecular Systems ${ }^{\circledR}$, Pleasanton, CA) using 55 cycles at $95^{\circ} \mathrm{C}$ for 2 seconds and $56^{\circ} \mathrm{C}$ for 15 seconds. HRM data were analyzed as described previously. ${ }^{16}$ Samples were classified as wildtype if they possessed the same nucleotide sequence as our reference strain or mutant if not.

Statistical analysis. A CRF data entry form was developed in Census and Survey Processing System ${ }^{\circledR}$ version 7.2 (U.S. Census Bureau, Suitland, MD) and validated by the Laboratory and Chemosensitivity team within the Benin NMCP. Four data entry officers were responsible for double entry and data cleaning in collaboration with the Benin NMCP. We analyzed data using SAS version 9.3 (SAS Institute, Cary, NC). Descriptive data are presented as percentages, means, standard deviation, median, and ranges, as appropriate.

The primary study endpoints were the day 28 uncorrected and genotype-corrected ACPR proportions by per-protocol analysis. ${ }^{5}$ For these, the denominator included only participants with ACPR or treatment failure and excluded those with loss to follow-up or protocol violation. For the genotype-corrected analysis, the numerator and denominator also excluded recurrences on or after day 4 classified as reinfections by genotyping. In addition, we calculated cumulative success rate, estimated by genotype-corrected Kaplan-Meier analysis, considering all participants until they were censored, that is, recrudescence, reinfections, withdrawal, loss of followup, and ACPR.

Ethical considerations. The study was reviewed and approved by the National Health Research and Ethics Committee before starting of the study (Protocol BEN/004/2018/ NMCP). The Associate Director for Science, Center for Global Health, Centers for Disease Control and Prevention (CDC) also reviewed this proposal and considered CDC staff to be nonengaged in this research activity (Project no. 2017-141). Before inclusion, written informed consent from a participant's legal guardian was obtained. In addition, consent could be withdrawn at any time from the study without causing harm to a participant's care.

\section{RESULTS}

Enrollment. Of the 301 patients visiting either health facility from October 2018 to February 2019 who screened positive for $P$. falciparum, 145 participants were enrolled, 115 in Bohicon and 30 in Kandi. Because of the low enrollment rate in Kandi, a second patient enrollment was undertaken for that site, and an additional 60 patients were admitted after screening additional 100 patients from June to September 2019
(Figure 2). All positive samples were confirmed as $P$. falciparum by PCR.

Baseline characteristics. Participant median ages for Bohicon and Kandi were 30 and 41 months, respectively (Table 1). About half of participants were male (51.3\% in Bohicon and $51.1 \%$ in Kandi). All participants had fever at the enrollment visit; mean temperature at enrollment was $38.7^{\circ} \mathrm{C}$ and $38.6^{\circ} \mathrm{C}$ for Bohicon and Kandi, respectively. These and other variables at enrollment are listed in Table 1.

Side effects and patient follow-up. Adverse events reported during $A L$ treatment included nausea, abdominal pain, and vomiting. Only one participant vomited after having received a dose of medication; that participant tolerated the repeat dose. No participant was lost to follow-up; therefore, the uncorrected per-protocol analysis considered 115 and 90 participants in Bohicon and Kandi, respectively.

The arithmetic mean of asexual parasite densities at baseline was 46,424 (range: 611-199,603) and 59,774 (range: 2,100-199,010) in Bohicon and Kandi, respectively (Table 2). The proportion of participants with asexual parasitemia declined from $11.3 \%$ to $0 \%$ from day 2 to 3 , respectively, in Bohicon, with similar decreases in Kandi $(3.4 \%$ to $1.1 \%$, respectively). Data on mean parasite densities during followup are listed in Table 2. Mean hemoglobin levels increased from $7.9 \mathrm{~g} / \mathrm{dL}$ on day 0 to $8.5 \mathrm{~g} / \mathrm{dL}$ and $9.4 \mathrm{~g} / \mathrm{dL}$ on days 14 and 28, respectively, in Bohicon. In Kandi, hemoglobin levels were $8.6 \mathrm{~g} / \mathrm{dL}, 9.0 \mathrm{~g} / \mathrm{dL}$, and $10.2 \mathrm{~g} / \mathrm{dL}$ on days 0,14 , and 28 , respectively. This increase in both sites was statistically significant $(P<0.001)$.

Therapeutic efficacy. In Bohicon, 10 of 115 patients had recurrences classified as treatment failures in the uncorrected analysis; six patients had LPF and four LCF. The uncorrected ACPR was $91.3 \%$ (95\% Cl: 84.6-95.8\%) in the per-protocol analysis and $91.3 \%$ (95\% Cl: 85.5-95.7\%) in the KaplanMeier analysis. On the basis of genotyping analysis, four of these were classified as recrudescences, and six were classifies as reinfections. Thus, the genotype-corrected ACPR considered four failures and 109 patients and was estimated at 96.3\% (95\% Cl: 90.9-99.0\%), and the corresponding Kaplan-Meier estimate was 96.3\% (95\% Cl: 92.0-99.0\%).

In the uncorrected analysis, three of 90 patients had treatment failures in Kandi: one with ETF and two with LCF. Uncorrected ACPR was $96.7 \%$ (95\% Cl: 90.6-99.3\%) in the per-protocol analysis and $96.7 \%(95 \% \mathrm{Cl}$ : 92.0-99.4\%) in the Kaplan-Meier analysis. The two patients with LCF in the uncorrected analysis were found to be of the same genotype of those of day 0 and were classified as recrudescences. Therefore, we did not exclude any patient for the genotypecorrected ACPR analysis, reaching a per-protocol ACPR of 96.7\% (95\% Cl: 90.6-99.3\%) and a corresponding KaplanMeier ACPR of $96.7 \%$ (95\% Cl: 92.0-99.4\%). Raw genotyping data are available in supplemental materials. Figure 3 shows the genotype-corrected survival curve for both study sites using day 28 ACPR as the outcome of interest.

Molecular markers of resistance. No C580Y mutation in the PfK13 gene was observed because all genotyped isolates had the wildtype C580 allele.

\section{DISCUSSION}

We found that $A L$ remains highly efficacious for treatment of uncomplicated $P$. falciparum malaria in two sites in Benin after 
Bohicon

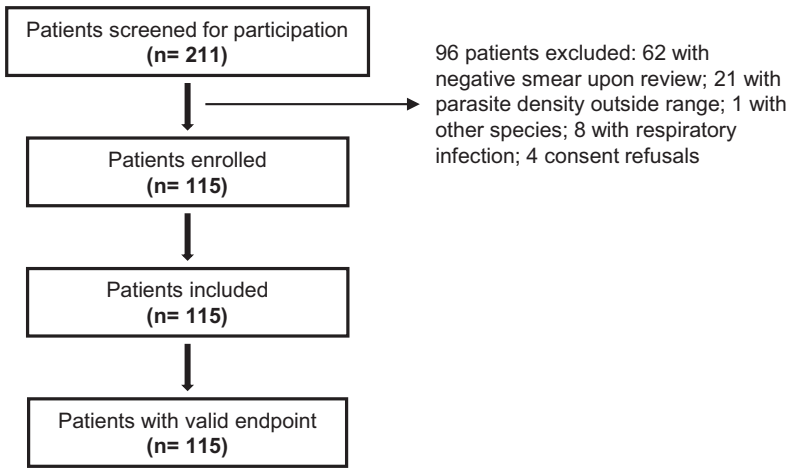

Kandi

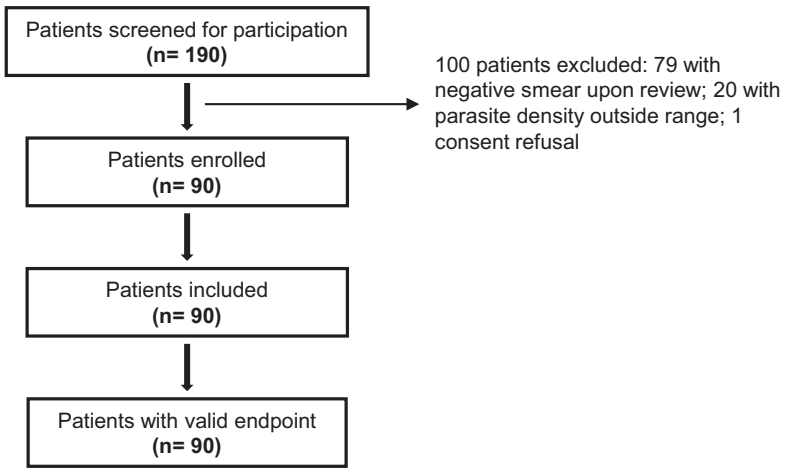

FlgURE 2. Screening, enrollment, and follow-up of study participants, Bohicon and Kandi, Benin, 2018-2019 ( $N=205)$. Note: In Kandi, 90 patients screened in the first enrollment phase in 2018 and 100 in the second in 2019 (see text for details).

16 years of $\mathrm{AL}$ use in Benin, with day 28 genotype-corrected ACPR of $96.3 \%$ and $96.7 \%$ in Bohicon and Kandi, respectively, in the per-protocol analysis. Day 3 parasitemia was not observed in Bohicon and was observed in only one (1.1\%) participant in Kandi, a low frequency that is consistent with good efficacy of the artemisinin component in this combination. Our study represents an effort by the Benin NMCP and its partners to monitor antimalarial efficacy in the country.

Our results are reassuring because the WHOrecommended threshold to consider a change in national antimalarial policy is an ACPR $<90 \% .{ }^{5,17}$ A prior study in Benin conducted in 2014 showed molecular-correct ACPR of $100 \%$ among children under 5 years of age treated with AL. ${ }^{18}$ The high AL efficacy might be related to the efforts to support the judicious use of antimalarials in Benin, with adherence to malaria testing results and to the combination therapy regimen itself, so that resistance to its components does not develop as it would if they were used as monotherapy or for insufficient number of days. There have been recent studies in neighboring Nigeria and Ghana with similarly high AL efficacy. ${ }^{11,19}$ However, other results from Ghana and Burkina Faso in West Africa and Angola in southern Africa have observed reduced efficacy of $A L$ in some regions, although those findings seem to be restricted to certain provinces. ${ }^{12,19-21}$ This variability in efficacy, with results close to the WHO threshold for treatment policy revision in some instances, speaks to the focal nature of antimalarial resistance and the importance of monitoring antimalarial efficacy across different areas in a country.

We noted a low hemoglobin level among our patients at enrollment, but that improved during follow-up. This finding is in line with studies in nearby Ghana where, after malaria treatment, hemoglobin levels improved rapidly. ${ }^{10,19}$ Prior studies in Benin have showed persistence of low levels of hemoglobin after malaria treatment. Our findings might signal recent improvements in child health programs addressing other causes of anemia in children, such as geohelminths. ${ }^{18}$ It also shows that malaria seems to be a main contributor to anemia in children and underscores the need for combating malaria as a means to improve childhood health markers.

Our study has a few limitations. We did not systematically collect information about adverse events associated with $\mathrm{AL}$ use, so we cannot accurately ascertain the frequency of these events. However, considering that only one patient in Kandi and none in Bohicon vomited after a treatment dose, we cautiously assume treatment tolerance was high, as in other studies in the region. ${ }^{22}$ We only evaluated the presence of the C580Y Pfk13 gene mutation. Other K13 mutations are now being observed at higher frequencies in some countries in Africa. Sanger sequencing in addition to other techniques for detection of Pfk13 gene mutations, such as amplicon deep sequencing, would have allowed for detection of other mutations in the gene associated with artemisinin resistance. ${ }^{23}$ We also did not evaluate other molecular markers, such as

TABLE 1

Characteristics of study participants at enrollment, Bohicon and Kandi, Benin, 2018-2019 (N=205)

\begin{tabular}{|c|c|c|}
\hline \multirow[b]{2}{*}{ Variable } & \multicolumn{2}{|c|}{ Value } \\
\hline & Bohicon & Kandi \\
\hline Patients enrolled, $n$ & 115 & 90 \\
\hline Age, months, median (range) & $30(6-58)$ & $41(6-59)$ \\
\hline Male, $n(\%)$ & $59(51.3)$ & $46(51.1)$ \\
\hline Weight (kg), mean (SD) & $12.3(2.8)$ & $14.0(3.3)$ \\
\hline Body temperature, ${ }^{\circ} \mathrm{C}$, mean (SD) & $38.7(0.9)$ & $38.6(0.8)$ \\
\hline Hemoglobin (g/dL), mean (SD) & $7.9(2.0)$ & $8.6(1.9)$ \\
\hline Asexual parasite density on day 0 , parasites $/ \mu \mathrm{L}$, arithmetic mean (range) & $46,424(611-199,603)$ & $59,774(2,100-199,010)$ \\
\hline Gametocytes presence on day $0, n(\%)$ & $1(0.9)$ & $0(0)$ \\
\hline
\end{tabular}


TABLE 2

Asexual parasite presence and density, and gametocyte presence by day of follow-up, Bohicon and Kandi, Benin, $2018-2019(N=205)$

\begin{tabular}{|c|c|c|c|c|c|c|}
\hline \multirow[b]{3}{*}{ Day } & \multicolumn{3}{|c|}{ Bohicon } & \multicolumn{3}{|c|}{ Kandi } \\
\hline & \multicolumn{2}{|c|}{ Asexual parasite presence and parasite density ${ }^{\star}$} & \multirow{2}{*}{$\begin{array}{c}\text { Gametocyte presence } \\
\text { Positive, } n(\%)\end{array}$} & \multicolumn{2}{|c|}{ Asexual parasite presence and parasite density ${ }^{\star}$} & \multirow{2}{*}{$\begin{array}{c}\text { Gametocyte presence } \\
\text { Positive, } n(\%)\end{array}$} \\
\hline & Positive, $n(\%)$ & Parasites $/ \mu \mathrm{L}$, arithmetic mean (range) & & Positive, $n(\%)$ & Parasites $/ \mu \mathrm{L}$, arithmetic mean (range) & \\
\hline 0 & $115(100 \%)$ & $46,424(611-199,603)$ & $1(0.9)$ & $90(100 \%)$ & 59,774. (2,100-199,010) & $0(0 \%)$ \\
\hline 2 & $13(11.3 \%)$ & $41.2(23-104)$ & $7(6.1 \%)$ & $3(3.4 \%)$ & $11,901.0(431-24,512)$ & $0(0 \%)$ \\
\hline 3 & $0(0 \%)$ & 0 & $0(0 \%)$ & $1(1.1 \%)$ & $82(82-82)$ & $0(0 \%)$ \\
\hline 7 & $0(0 \%)$ & 0 & $0(0 \%)$ & $0(0 \%)$ & 0 & $0(0 \%)$ \\
\hline 14 & $0(0 \%)$ & 0 & $0(0 \%)$ & $0(0 \%)$ & 0 & $0(0 \%)$ \\
\hline 21 & $6(5.21 \%)$ & $57,201.2(5,804-23,025)$ & $1(0.9 \%)$ & $2(2.22 \%)$ & $246,512.5(233,025-260,000)$ & $0(0 \%)$ \\
\hline 28 & $2(1.7 \%)$ & $36,975.0(2,830-71,436)$ & $2(1.7 \%)$ & $0(0 \%)$ & 0 & $0(0 \%)$ \\
\hline
\end{tabular}

$P$. falciparum multidrug-resistant 1 (pfmdr1) gene, associated with decreases in lumefantrine susceptibility in some studies, which would have allowed us to also infer presence of resistant markers to the partner drug of the ACT used in Benin. ${ }^{24,25}$ Considering seasonal chemoprevention with sulfadoxinepyrimethamine and amodiaquine is used in parts of Benin, resistance markers to these drugs should also be considered for samples collected as part of in vivo studies. However, the lack of the genetic information does not hamper our conclusions because genotype corrected ACPR remains the main outcome for TESs.

We recognize TESs are the gold-standard methodology for antimalarial efficacy monitoring and policy decision-making. ${ }^{5}$ Conducting TESs at the required intervals - every 2 years - is not a trivial task for malaria control programs because it requires allocating resources, personnel, and commitment from multiple partners, especially for required genetic testing. Benin NMCP has been able to conduct four rounds of TESs from 2007 to 2018; results from two have been publicly disseminated. The last two rounds of TESs also included genotyping correction. Obstacles to timely TES data analysis and reporting should be evaluated and addressed so malaria

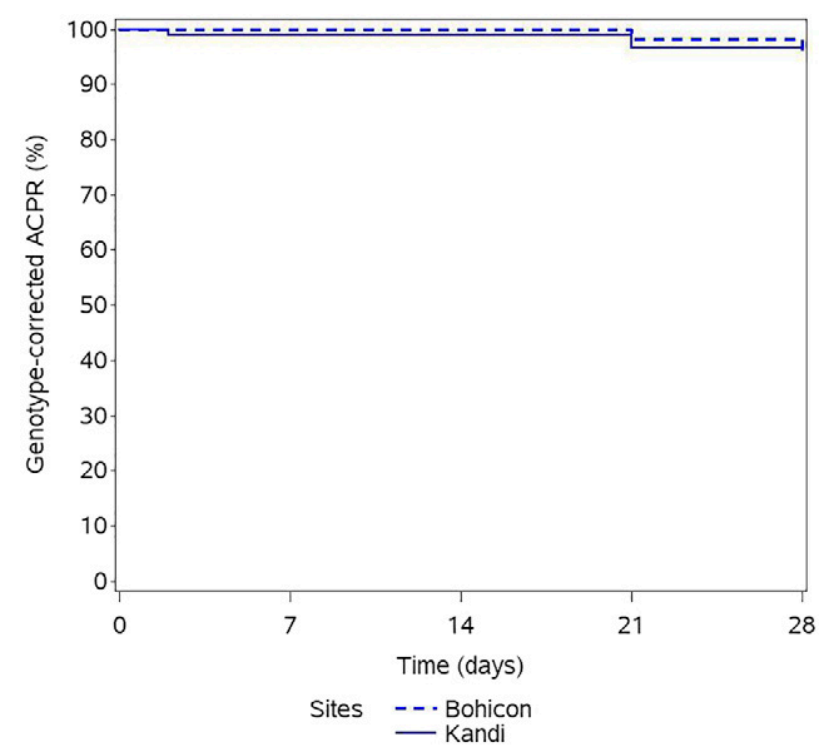

FIGURE 3. Genotype-corrected day 28 adequate clinical and parasitological response (ACPR) survival curve per site, Bohicon and Kandi, Benin $(N=205)$. This figure appears in color at www.ajtmh.org. control programs can consider changes to their treatment policies as early as warranted.

We recognize the importance of collecting antimalarial efficacy from different regions of a country, as efficacy can vary and pockets with distinct resistance profiles may be present. ${ }^{19,26}$ As countries move forward to regular and sustainable collection of antimalarial efficacy and resistance information, it is important to find the right balance on selecting sites for longitudinal monitoring and also improve in-country capacity to implement and supervise TES and perform molecular tests for genotyping and resistance markers. This will allow for early detection of antimalarial resistance and support evidence-based antimalarial policies.

In summary, our study found evidence of continued high efficacy of $A L$ in Benin, which supports its ongoing use as first-line therapy for uncomplicated $P$. falciparum malaria in the country. However, regular in vivo monitoring and molecular surveillance of mutations in genes associated with artemisinin (Pfk13) and partner drug resistance in the region are recommended every 2 years per WHO guidelines to inform antimalarial treatment policies.

Received January 22, 2021. Accepted for publication April 19, 2021.

Published online July 12, 2021.

Note: Supplemental material appears at www.ajtmh.org.

Acknowledgments: We thank the participants and their caregivers who kindly agreed to the 28-day follow-up; study personnel who made it possible to enroll and follow patients; and the staff at Bohicon and Kandi health centers for their collaboration and support. We are also indebted to all the team of the Molecular Biology and Genomics Laboratory at Aristide Hospital DANTEC in Dakar, Senegal, for their welcome and their technical assistance and supervision of the molecular biology component, specially Mouhamad Sy, Tolla Ndiaye, and Amy Gaye. Thank you to Dr. Alexandre Macedo for his technical review of the manuscript. Finally, many thanks to Dr. Cyriaque Degbe and Dr. Razac Osse for their technical review of the study protocol as part of the ethical approval process in Benin.

Financial support: Funding for this study was partially provided by the U.S. Agency for International Development through the President's Malaria Initiative.

Disclaimer: The funding sources for this study had no role in study design, data collection, analysis, or interpretation. The opinions expressed herein are those of the authors and do not necessarily reflect the views of the Benin National Malaria Control Program, U.S. Agency for International Development, or the Centers for Disease Control and Prevention.

Authors' addresses: Augustin Kpemasse, Programme National de Lutte contre le Paludisme, Laboratory Service and Chemosensitivity, Cotonou, Benin, E-mail: kpemasseaugustin@yahoo.fr. Fortune Dagnon, USAID, Benin, Cotonou, E-mail: lecuredars@yahoo.fr. 
Ramani Saliou, Alexis Sacca Yarou Maye, Cyriaque Dossou Affoukou, Programme National de Lutte contre le Paludisme, MS, Cotonou, Benin, E-mails: ramani.saliou@yahoo.fr, yarou_alexis@yahoo.fr, and moiacdm@yahoo.fr. Alassane Zoulkaneri, Ministry of Health Benin, Kandi Health Center, Kandi, Benin, E-mail: alphazoul@yahoo.fr. Blaise Guézo-Mévo, Hopital de zone, Département de senté du Mono-Couffo, Comé, Benin, E-mail: guemebla@yahoo.fr. Leah F. Moriarty, Centers for Disease Control and Prevention Center for Global Health, Malaria Branch, Atlanta, GA, E-mail: wvp4@cdc.gov or Imoriarty@cdc.gov. Yaye D. Ndiaye, Awa Bineta Deme, and Daouda Ndiaye, Univerté Cheikh Anta Diop, ParasitologieMycologie, Dakar, Senegal, E-mails: ydndiaye@gmail.com, deme. awa@gmail.com, and dndiaye@hsph.harvard.edu. Mamane Nassirou Garba, Hospital DANTEC, Molecular Biology and Genomics Laboratory, Dakar, Senegal, E-mail: nassirou.garba@gmail.com. Aurore Ogouyemi Hounto, Programme National de Lutte contre le Paludisme, Directorate, Cotonou, Benin, E-mail: aurorefel@yahoo.fr.

\section{REFERENCES}

1. World Health Organization, 2020. World Malaria Report 2020: 20 Years of Global Progress and Challenges. Geneva, Switzerland: WHO. Available at: https://www.who.int/publications/i/item/ 9789240015791.

2. Santé Mdl, 2019. Annuaire des statistiques sanitaires. Cotonou, Benin: Santé Mdl, 144.

3. Haldar K, Bhattacharjee S, Safeukui I, 2018. Drug resistance in Plasmodium. Nat Rev Microbiol 16: 156-170.

4. World Health Organization, 2015. Guidelines for the Treatment of Malaria. Available at: https://www.who.int/docs/default-source/ documents/publications/gmp/guidelines-for-the-treatment-ofmalaria-eng.pdf?sfvrsn=a0138b77_2. Accessed April 6, 2021.

5. World Health Organization, 2009. Methods for Surveillance of Antimalarial Drug Efficacy. Geneva, Switzerland: WHO. Available at: https://apps.who.int/iris/bitstream/handle/10665/ 44048/9789241597531_eng.pdf?sequence=1\&isAllowed=y.

6. World Health Organization, 2015. Status Report on Artemisinin Resistance. Geneva, Switzerland: WHO. Available at: https:// www.who.int/malaria/publications/atoz/status-rep-artemisininact-resistance-sept2015.pdf. Accessed April 6, 2021.

7. Santé Mdl, 2017. Directives Nationales de Prise en Charge des Cas de Paludisme. Cotonou, Benin: Paludisme PNdLcl, 6.

8. World Health Organization, 2011. Global Plan for Artemisinin Resistance Containment. Geneva, Switzerland: WHO. Available at: https://www.who.int/malaria/publications/atoz/ 9789241500838/en/. Accessed August 10, 2020

9. Ogouyèmi-Hounto $A$, Azandossessi $C$, Lawani $S$, Damien $G$, de Tove YSS, Remoue F, Gazard DK, 2016. Therapeutic efficacy of artemether-lumefantrine for the treatment of uncomplicated falciparum malaria in northwest Benin. Malar J 15: 1-8.

10. Abuaku B, Duah N, Quaye L, Quashie N, Malm K, Bart-Plange C, Koram K, 2016. Therapeutic efficacy of artesunateamodiaquine and artemether-lumefantrine combinations in the treatment of uncomplicated malaria in two ecological zones in Ghana. Malar J 15: 6.

11. Ebenebe JC, et al., 2018. Efficacy of artemisinin-based combination treatments of uncomplicated falciparum malaria in underfive-year-old Nigerian children ten years following adoption as first-line antimalarials. Am J Trop Med Hyg 99: 649-664.
12. Gansané A, et al., 2021. Anti-malarial efficacy and resistance monitoring of artemether-lumefantrine and dihydroartemisininpiperaquine shows inadequate efficacy in children in Burkina Faso, 2017-2018. Malar J 20: 1-12.

13. Borre MB et al., 1991. Primary structure and localization of a conserved immunogenic Plasmodium falciparum glutamate rich protein (GLURP) expressed in both the preerythrocytic and erythrocytic stages of the vertebrate life cycle. Mol Biochem Parasitol 49: 119-131.

14. Fenton B, Clark JT, Khan CM, Robinson JV, Walliker D, Ridley R, Scaife JG, McBride JS, 1991. Structural and antigenic polymorphism of the 35- to 48-kilodalton merozoite surface antigen (MSA-2) of the malaria parasite Plasmodium falciparum. Mol Cell Biol 11: 963-971.

15. Cattamanchi A, Kyabayinze D, Hubbard A, Rosenthal PJ, Dorsey $G, 2003$. Distinguishing recrudescence from reinfection in a longitudinal antimalarial drug efficacy study: comparison of results based on genotyping of msp-1, msp-2, and glurp. Am J Trop Med Hyg 68: 133-139.

16. Daniels R, Hamilton EJ, Durfee K, Ndiaye D, Wirth DF, Hartl DL, Volkman SK, 2015. Methods to increase the sensitivity of high resolution melting single nucleotide polymorphism genotyping in malaria. J Vis Exp 105: 52839.

17. Vestergaard LS, Ringwald P, 2007. Responding to the challenge of antimalarial drug resistance by routine monitoring to update national malaria treatment policies. Am J Trop Med Hyg 77: 153-159.

18. Ogouyemi-Hounto A, Azandossessi C, Lawani S, Damien G, de Tove YS, Remoue F, Kinde Gazard D, 2016. Therapeutic efficacy of artemether-lumefantrine for the treatment of uncomplicated falciparum malaria in northwest Benin. Malar J 15: 37.

19. Abuaku B, Duah N, Quaye L, Quashie N, Koram K, 2012. Therapeutic efficacy of artemether-lumefantrine combination in the treatment of uncomplicated malaria among children under five years of age in three ecological zones in Ghana. Malar $J$ 11: 388.

20. Davlantes E et al., 2018. Efficacy and safety of artemetherlumefantrine, artesunate-amodiaquine, and dihydroartemisininpiperaquine for the treatment of uncomplicated Plasmodium falciparum malaria in three provinces in Angola, 2017. Malar $J$ 17: 144.

21. Plucinski MM et al., 2015. Efficacy of artemether-lumefantrine and dihydroartemisinin-piperaquine for treatment of uncomplicated malaria in children in Zaire and Uige Provinces, angola. Antimicrob Agents Chemother 59: 437-443.

22. Niare $\mathrm{K}$ et al., 2016. In vivo efficacy and parasite clearance of artesunate + sulfadoxine-pyrimethamine versus artemetherlumefantrine in Mali. Am J Trop Med Hyg 94: 634-639.

23. Talundzic $E$ et al., 2018. Next-generation sequencing and bioinformatics protocol for malaria drug resistance marker surveillance. Antimicrob Agents Chemother 62: e02474-17.

24. de Laurent ZR, Chebon LJ, Ingasia LA, Akala HM, Andagalu B, Ochola-Oyier LI, Kamau E, 2018. Polymorphisms in the K13 gene in Plasmodium falciparum from different malaria transmission areas of Kenya. Am J Trop Med Hyg 98: 1360-1366.

25. Ljolje D et al., 2018. Prevalence of molecular markers of artemisinin and lumefantrine resistance among patients with uncomplicated Plasmodium falciparum malaria in three provinces in Angola, 2015. Malar J 17: 84.

26. Fancony C, Brito M, Gil JP, 2016. Plasmodium falciparum drug resistance in Angola. Malar J 15: 74 